\title{
Implementation of Multiparametric Magnetic Resonance Imaging Technology for Evaluation of Patients with Suspicion for Prostate Cancer in the Clinical Practice Setting
}

Paras H. Shah, MD ${ }^{1}$; Vinay R. Patel, BS ${ }^{2}$; Daniel M. Moreira, $\mathrm{MD}^{3}$; Arvin K. George, $\mathrm{MD}^{4}$; Manaf Alom, MBBS ${ }^{1}$; Zachary Kozel, MD ${ }^{5}$; Vidhu Joshi, BS ${ }^{1}$; Eran Ben-Levi, MD ${ }^{6}$; Robert Villani, MD ${ }^{6}$; Oksana Yaskiv, $\mathrm{MD}^{7}$; Louis R. Kavoussi, MD5 Manish Vira, $\mathrm{MD}^{5}$; Carl O. Olsson, $\mathrm{MD}^{8}$; Ardeshir

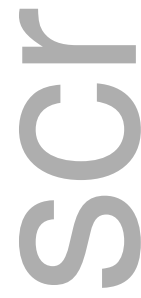
R. Rastinehad, $\mathrm{DO}^{2}$

1. Department of Urology, Mayo Clinic, Rochester, $M N$

2. Department of Urology and Radiology, Icahn School of Medicine at Mount Sinai, New York, NY 3. Department of Urology, University of Illinois at Chicago, Chicago, IL 4. Department of Urology, University of Michigan, Ann Arbor, MI

5. Department of Urology, Smith Institute for Urology, Northwell Health, New Hyde Park, NY 6. Department of Radiology, Hofstra Northwell School of Medicine, New Hyde Park, NY 7. Department of Pathology, Hofstra Northwell School of Medicine, New Hyde Park, NY

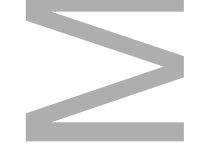
8. Integrated Medical Professionals, NY

Corresponding Author: Ardeshir R. Rastinehad

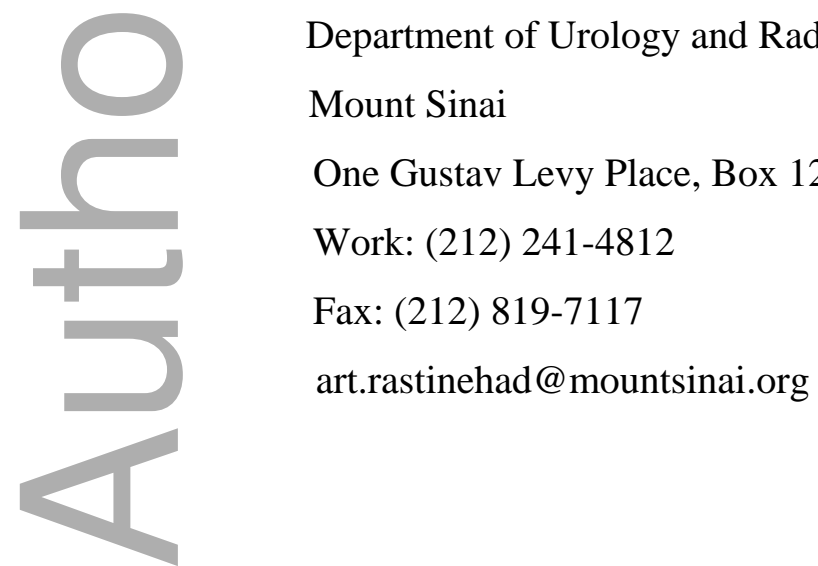

\section{Manuscript Word Count: 3641}

This is the author manuscript accepted for publication and has undergone full peer review but has not been through the copyediting, typesetting, pagination and proofreading process, which may lead to differences between this version and the Version of Record. Please cite this article as doi: $\underline{10.1111 / \text { bju. } 14515}$

This article is protected by copyright. All rights reserved 
Key Words: Magnetic Resonance Imaging, Prostate Cancer, Biopsy

Disclosures/Conflicts of Interest: None
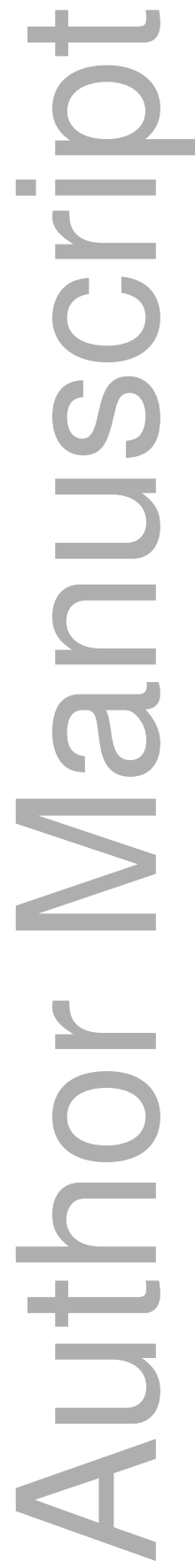

This article is protected by copyright. All rights reserved 


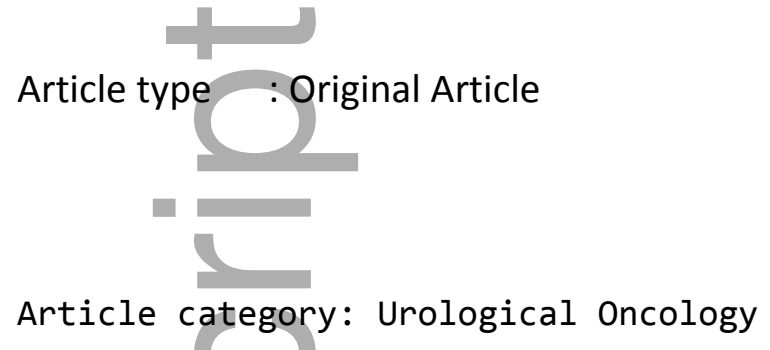

\section{Introduction}

Multi-parametric magnetic resonance imaging (mpMRI) has emerged as a promising tool in the detection of clinically significant prostate cancer $(\mathrm{csPCa}){ }^{1-3}$ Limitations associated with the recognition of low-grade, low volume pathology can be considered a strength, as this allows for selection of patients with high-risk disease features, for which mpMRI exhibits remarkable specificity. ${ }^{2,4}$ In view of current criticisms by the United States Preventative Services Task Force (USPSTF) that prostate specific antigen (PSA) screening invites unnecessary intervention and treatment, the use of mpMRI as an adjunct to PSA creates opportunity to consider prostate biopsy primarily for those patients with highest suspicion for clinically significant disease. ${ }^{5,6}$

Although level 1 evidence supports the ability of mpMRI to preferentially detect higherrisk disease in patients with elevated PSA, results from existing trials are generated in highly idealized settings where comparison to cohorts evaluated with PSA alone involves the universal biopsy of all study patients for purposes of sensitivity and specificity analyses. ${ }^{1,6}$ In this respect, our understanding of the advantages of MRI to help guide biopsy decision in men with elevated PSA remains theoretical as no large-scale study to date evaluates its performance when integrated into a true clinical practice setting, where decisions for biopsy are not necessarily driven by defined protocols, but often based on personalized patient assessments.

Herein, we investigated how the implementation of mpMRI and MR/US fusion-guided technology in two large academic and community urology practices within the United States influenced patterns of prostate biopsy and cancer detection in a large cohort of men who presented with elevated PSA. With the USPSTF guidelines highlighting the inherent weaknesses of PSA-based screening, we contend that use of mpMRI to triage patients with elevated PSA 
would address these legitimate concerns in true clinical practice by decreasing biopsy rates, minimizing risks inherent in indiscriminate systematic prostate sampling, and enabling the preferential detection of csPCa when compared to men in whom biopsy decisions are guided by conventional clinical metrics centered around PSA.,7

\section{Methods}

Following Institutional Review Board approval, data were retrospectively collected from patients (aged $\geq 18$ years) referred for elevated PSA between June 2011 and November 2014 to two large independent academic-community practices in New York City and Long Island, NY (Smith Institute for Urology and Integrated Medical Professionals). The study population was divided into MRI and PSA-only groups based on whether patients underwent mpMRI of the prostate at any point during the specified accrual period. All patients were counseled on the use of mpMRI as a secondary test in the evaluation of elevated PSA. Reasons for MRI not being performed included lack of patient desire, medical contraindication to MRI, physician decision to defer imaging, or inability to obtain insurance approval for the MRI study (Supplementary Table 1). Both cohorts were followed longitudinally until October 2016. Patients with prior prostate cancer (PCa) history, history of prostate MRI prior to initial visit during enrollment period, or $<1$-year follow-up were excluded. Patients in the PSA-only group who had negative biopsy during the accrual period and underwent subsequent mpMRI during follow-up comprised a distinct cohort and were not included in the primary analysis; this was done to enable a more representative description of biopsy practice and cancer-detection in a population evaluated purely based conventional clinical metrics without MRI imaging.

Variables evaluated included age, race, family history of $\mathrm{PCa}$, prior negative prostate biopsy, digital rectal exam (DRE), baseline PSA, and prostate volume. Subjects undergoing MRI were stratified into two groups (positive or negative) based on the presence of suspicion lesions. Suspicious findings were defined based on a 5-point Likert scale as any lesions on MRI with Simplified Qualitative System (SQS) score ranging between 1 and 5, with a SQS score of $\leq 2$ corresponding to a negative MRI and an SQS score between 3 and 5 corresponding to a positive MRI and prompting fusion biopsy. ${ }^{8}$ The SQS scoring system has been previously described and was utilized as implementation of our institutional MRI program predated publication of the PI- 
RADS classification. Nevertheless, we have demonstrated similar overall risk stratification of MRI lesions based on SQS and PI-RADS V2 scoring systems. ${ }^{8}$

Patients were evaluated for use of post-MRI or post-PSA prostate biopsy in MRI and PSA-only cohorts, respectively, as well as biopsy pathology when applicable. Individuals with positive MRI were offered MRI/Transrectal ultrasound (TRUS) fusion-guided biopsy of suspicious lesions in addition to standard 12-core biopsy, performed during the same setting. Fusion biopsy was performed using the UroNav MRI/TRUS (end-fire iU22 ultrasound, Philips Healthcare, Netherlands) fusion guided prostate biopsy system (Invivo, Gainesville, FL). Two biopsy cores were obtained from each lesion; one in the axial and the other in the sagittal plane. After targeted biopsy was performed of the suspicious lesion(s), the UroNav workstation where the MRI was processed was turned off and a standard 12-core systematic TRUS-guided prostate biopsy was subsequently performed.

Clinically significant PCa was defined as any Gleason score $\geq 7$ on fusion or standard 12core TRUS biopsies, Gleason 6 with a lesion volume $>0.5 \mathrm{~cm}^{3}$ volume on MRI, or Gleason 6 with $>2$ cores positive and/or $>50 \%$ of any core involved with cancer on biopsy per Epstein's criteria. $^{9}$

Treating providers were the same for patients in both study groups. Biopsy was performed based on aggregate analysis of MRI findings (in the MRI cohort) and clinical variables including PSA, DRE, prior history of biopsy, race, and family history as well as patient desire. A 3-Tesla Verio® MRI (Siemens, Germany) was performed using a 16-channel cardiac coil (Sense, Invivo®) and an endorectal coil (BPX-30, Medrad®, Pittsburgh, PA) filled with PFC-770 (3M®), St. Paul, MN). ${ }^{8}$ Sequences obtained included tri-planar T2-weighted imaging, axial diffusion-weighted imaging (b values 0, 50, 500, 1000, 1500, and 2000) with apparent diffusion coefficient mapping (b-values 0, 50, 500, 1000, 1500), and dynamic contrast-enhanced imaging. All mpMRI were read prospectively by experienced genitourinary radiologists and scored with a five-point Likert scale (SQS score) with sequence specific information that could be used to calculate other scoring systems that may arise (PI-RADSv1). ${ }^{8}$ Patients with a negative MRI who did not undergo biopsy were followed with PSA and/or interval imaging.

Descriptive statistics are presented as counts and percentages for categorical variables and as means and standard deviations for continuous variables. The association of patient and disease characteristics by study group (i.e. MRI vs. PSA-only) was analyzed with Student's t-test 
for continuous data and chi-square test for categorical variables in univariable analysis. Primary outcomes included rate of prostate biopsy in MRI versus PSA-only groups as well as overall PCa detection among all-comers and those who had a post-MRI/PSA prostate biopsy. Secondary outcomes included presence of Gleason score 6, 7, and 8-10 cancers as well as csPCa among biopsied patients in the MRI versus PSA-only groups. Multivariable analysis of PCa detection variables was conducted with multinomial logistic regressions adjusting for the covariates age, race, family history of PCa, DRE, history of previous negative biopsy, and baseline PSA. All analyses were two-tailed and performed using Stata 14.2 (College Station, TX). A P<0.05 was considered statistically significant.

\section{Results}

Between June 2011 and November 2014, 2073 patients without history of PCa presented to both urology practices for evaluation of elevated PSA. 167 patients evaluated with PSA only during the accrual period underwent subsequent mpMRI during follow-up and were not included in the analysis. 42 patients who had an MRI of the prostate prior to accrual and 56 patients with follow-up < 1 year were excluded. The final study cohort consisted of 1020 patients and 788 patients in MRI and PSA-only arms, respectively (Supplementary Figure). Patient demographics and clinical characteristics are delineated in Table 1. Prior negative biopsy (59.9\% vs. 32.6\%, $\mathrm{P}<0.001)$ was significantly more frequent among those undergoing MRI. No differences in baseline PSA, DRE, family history and race were appreciated between cohorts. Of the 1020 patients undergoing MRI, 452 (44.3\%) patients had a positive MRI. The distribution of suspicion scores for observed lesions is presented in Supplementary Table 2.

Over a median follow-up of 3.1 years, a total 465 (45.6\%) and 442 (56.1\%) patients underwent biopsy in the MRI and in PSA-only groups, respectively, corresponding to an $18.7 \%$ decrease in the proportion of patients undergoing biopsy in the MRI group ( $\mathrm{P}<0.001$, Table 2). Biopsy was performed in $378(83.6 \%)$ patients with positive MRI versus $87(15.3 \%)$ with negative MRI. Median follow-up among patients with a negative MRI who did not undergo biopsy was 3.6 years. Repeat mpMRI was performed in 35 (7.3\%) patients, with the study having remained negative in all patients. One patient had undergone biopsy from this cohort and was negative for PCa. Median follow-up for patients in the PSA-only group who did not undergo biopsy or had negative biopsy was 3.4 years

This article is protected by copyright. All rights reserved 
Among biopsied patients, overall $\mathrm{PCa}(56.8 \%$ vs. $40.7 \%, \mathrm{P}<0.001)$ and csPCa detection (47.3\% vs. $31.0 \%, \mathrm{p}<0.001)$ were significantly higher in the MRI versus PSA-only group, corresponding to increased detection of GS 7 lesions $(52.3 \%$ vs. $40.6 \%, \mathrm{P}=0.018)$ and decreased detection of GS 6 disease ( $27.7 \%$ vs. $40 \%, \mathrm{P}=0.018$, Table 2$)$. In multivariable analysis, the odds of overall $\mathrm{PCa}(\mathrm{OR}=1.74,95 \% \mathrm{CI}=1.29-2.35, \mathrm{P}<0.001)$ and $\mathrm{csPCa}$ detection $(\mathrm{OR}=2.04$, $95 \% \mathrm{CI}=1.48-2.80, \mathrm{P}<0.001)$ were significantly higher in the MRI compared to PSA-only group (Table 3).

Biopsy rates and pathologic findings stratified by MRI result and SQS scores are presented in Supplementary Table 2. Incidence of csPCa within lesions of SQS score 3, 4, and 5 was $27.5 \%, 70.9 \%$, and $98.5 \%$, respectively. Among the 87 patients with negative MRI who underwent biopsy, cancer was detected in $15(17.2 \%)$ cases, with $8(9.2 \%)$ having qualified as clinically significant disease.

$324(85.7 \%)$ of 378 patients with a positive MRI underwent combined fusion with 12core systematic TRUS biopsy (Table 4 and Supplementary Table 3). Overall cancer detection rate in this group was $65.7 \%$, including $54.9 \%$ for fusion biopsies and $54.3 \%$ for 12 -core systematic biopsies. Detection of csPCa, however, was significantly higher on targeted fusion biopsy than on 12-core systematic TRUS biopsy (50.3\% vs $41.4 \%, \mathrm{p}=0.022$, Table 4), which corresponded to increased detection of GS 7 lesions (60.5\% vs. 47.7\%, $\mathrm{P}=0.009)$. Of the 14 clinically significant tumors missed on fusion biopsy, seven were GS 6 tumors and seven were GS 7 tumors.

\section{Discussion}

Improved understanding of the biologic heterogeneity of PCa has unveiled the gross over-detection and often unnecessary treatment of clinically insignificant cancers through widely utilized PSA-based screening practices. ${ }^{5,7}$ Consensus statements issued by the USPSTF arguing against the universal use of PSA would theoretically diminish morbidity related to diagnosis of low-risk PCa, but also leave vulnerable a population of patients with higher-risk pathologies shown to benefit from primary intervention. ${ }^{5,7,10-14}$ This dilemma highlights the need for a more refined approach to the management of patients with elevated PSA that selectively identifies patients with clinically significant disease. 
Our study results offer a real-world interpretation of how integration of MRI technology in the triage of patients with elevated PSA influences patterns of prostate biopsy and cancer detection compared to PSA alone. We show in the clinical practice setting that utilization of MRI as a risk-assessment tool significantly reduces the use of prostate biopsy when compared to clinical decision-making based on PSA alone. At 3.1-year follow-up, an $18.7 \%$ reduction in biopsy rate among men evaluated with MRI was observed in the context of biopsy being performed in only $15.3 \%$ of men with negative MRI compared to $83.6 \%$ of men with positive MRI. Moreover, despite the reduction in biopsy rate, PCa detection increased by $39.4 \%$ in the MRI cohort, which corresponded with a $52.6 \%$ increase in detection of clinically significant pathology. Benefit derived from MRI was seen to extend beyond the identification of men with a positive study, but also involved the ability to perform targeted biopsy of suspicious lesions as incidence of csPCa was appreciably higher among patients receiving combined fusion with systematic 12-core TRUS biopsy than in patients with positive MRI who only underwent nontargeted 12-core TRUS biopsy.

Several studies lend support to our findings, albeit in the context of highly idealized trial scenarios. In a pilot study nested within the Goteborg randomized screening trial, use of targeted biopsy alone in 124 men with a suspicious lesion on pre-biopsy MRI would reduce biopsy rates by at least $33 \%$ while leading to an $48 \%$ increase in detection of csPCa when compared to systematic biopsy based on elevated PSA value alone. ${ }^{15}$ Similarly, in a prospective assessment of MRI use in the setting of elevated PSA, the PROMIS trial presented two hypothetical models for patient evaluation: one based on standard practice of TRUS biopsy in men with elevated PSA, and another utilizing MRI as a triage tool, reserving biopsy for men with suspicious imaging lesions. Comparison of both pathways revealed a significantly higher positive predictive value for the MRI-based approach, as the incidence of csPCa in standard TRUS biopsy and MRI biopsy cohorts was $19 \%$ and $51 \%$, respectively. ${ }^{1}$ Most recently, Kasivisvanathan et al randomized 500 patients at risk of PCa to TRUS biopsy or MRI/ultrasound-guided fusion biopsy and determined that using MRI prior to biopsy was superior to standard TRUS biopsy with regards to increased detection of csPCa and reduced detection of clinically-insignificant disease. ${ }^{6}$

Our study places the findings of the PROMIS and PRECISION trials into more practical context, illustrating in a contemporary practice setting how implementation of MRI into the triage algorithm for elevated PSA would indeed facilitate preferential identification of patients 
with aggressive disease while mitigating over-diagnosis of insignificant pathology. Fundamental assumptions of both the PROMIS and PRECISION trials predicating the superiority of an MRIbased risk assessment are that firstly, all patients presenting with elevated PSA would be candidates for MRI and that secondly, TRUS biopsy would be performed in all patients with elevated PSA, both of which are not representative of true clinical practice within the United States; in fact, among 788 patients in our study's PSA-only group, only 56\% went on to receive biopsy at a median 3.1 year follow-up. Nevertheless, despite the selective use of biopsy even in the PSA-only cohort, we still observed a significantly higher positive predictive value for cancer detection with the MRI-based approach, with csPCa detection having increased over 50\% when compared to biopsy based on PSA alone. Also notable is the higher detection rate for csPCa in the MRI cohort despite greater incidence of prior negative biopsy, further supporting the sensitivity of MRI for aggressive disease phenotypes compared with PSA-driven systematic biopsy.

The benefit we observed to performing targeted biopsy of suspicious lesions is consistent with what is reported in the contemporary literature. ${ }^{2,16,17}$ A systematic review evaluating the role of MRI with and without fusion technology demonstrated that while overall PCa diagnosis rates were similar between targeted biopsy and non-targeted 12-core TRUS biopsy, use of targeted biopsy significantly increased detection of aggressive subtypes. ${ }^{16}$ Similarly, addition of standard TRUS biopsy to targeted biopsy in patients with positive MRI was shown to be of limited utility by Siddiqui et al in a landmark prospective trial evaluating MR-fusion biopsy in 1003 patients with clinical suspicion for PCa. Although the combination of 12-core TRUS biopsy and targeted biopsy increased diagnosis of PCa by $10 \%$ in their study cohort, less than half would qualify as clinically significant with only $3 \%$ representing intermediate or high-risk disease. ${ }^{2}$

A diagnostic platform integrating MRI as a secondary test in men with elevated PSA would be premised on omission of biopsy in the absence of suspicious imaging lesions, a practice which raises concern for the missed detection of clinically significant disease in patients with elevated PSA but negative MRI. Previous attempts to discern the negative predictive value (NPV) of MRI are limited by selection biases inherent in the study of radical prostatectomy cohorts, largely skewing disease detection rates. A more accurate assessment of MRI sensitivity for csPCa is provided by the PROMIS trial, as transperineal mapping biopsy of men with elevated PSA without a diagnosis of PCa represents a more balanced pathologic reference 
standard. Using Gleason score $\geq 4+3$ as a primary definition for csPCa, Ahmed et al observed that $89 \%$ of men with negative MRI had absent or indolent pathology on transperineal biopsy, translating to a NPV significantly higher than that seen after standard 12-core TRUS biopsy when performed for elevated PSA. ${ }^{1}$ Even when considering a more inclusive definition for csPCa (GS $\geq 3+4$ or cancer core length $\geq 4 \mathrm{~mm}$ ), which is more in line with our study criteria, the NPV of MRI (72\%) remained significantly greater than 12-core TRUS biopsy (65\%), indicating that while MRI is not perfect at excluding the presence of clinically significant disease, it does demonstrate incremental benefit over the current standard practice of non-targeted systematic TRUS biopsy. ${ }^{1}$

Although our series may underestimate the risk for missed detection of csPCa in the MRI cohort given that biopsy was not performed in all patients with negative MRI, there are several factors that allow us to infer a relatively favorable NPV from the current literature. ${ }^{1,17}$ All patients in our series received high-quality imaging using a 3-Tesla magnet with an endorectal coil, meeting the standards of the American College of Radiology as outlined in the PI-RADS V2 module. ${ }^{18}$ Furthermore, biopsy was only performed by experienced providers well beyond their initial learning curve. This is in contrast to the PROMIS and PRECISION trials, in which a favorable negative predictive value and a high level of confidence in a negative MRI result was achieved despite use of 1.5-Tesla magnet without endorectal coil and biopsy being performed by providers with varying level of experience. ${ }^{1,6}$ Thus, the high- quality of MRI performed in the present study would lead us to believe that our rate of missed csPCa in negative MRI patients is not significantly different than what is established in the current literature. Nevertheless, as biopsy was not performed in all patients with negative MRI, broader conclusions regarding the NPV of MRI in men with elevated PSA cannot be drawn from this study.

Several limitations of our study should be noted. Biopsy in both study cohorts was performed at the discretion of individual practitioners after evaluation of imaging and patientspecific clinical characteristics rather than based on set criteria, potentially contributing to differences in biopsy rates and PCa detection. However, our study objective was to capture this variability that often exists in true clinical practice and demonstrate the utility of mpMRI as a risk-assessment tool within this framework. Reasons for MRI having not been performed were multifactorial and included lack of insurance approval as well as patient refusal. Inability to obtain insurance approval for MRI may have been a reflection of underlying 
socioeconomic differences between the PSA-only and MRI groups, which may have in turn influenced observed differences in biopsy and cancer detection rates between groups. Similarly, patients who refused MRI may have done so because of an inherent inclination to proceed with biopsy in the setting of elevated PSA; this may have contributed to increased biopsy rates in the PSA-only group. A higher prior negative biopsy rate was observed in the MRI arm, which is not entirely unexpected given its current use primarily in this setting. Although this may have influenced biopsy practice patterns, higher cancer detection rates in this cohort speaks to a strength of MRI as a tool to capture disease which may have otherwise been missed using conventional PSA-based screening. Furthermore, our study offers real-world perspective on the feasibility of integrating MRI technology into the contemporary clinical practice setting, where the universal use of MRI in the evaluation of patients with elevated PSA is currently not standard practice.

The negative stigma associated with PSA screening based on the USPSTF recommendations may have also influenced the decision to pursue biopsy among patients presenting with elevated PSA and explain in part differences between study groups. Nevertheless, any impact of the USPSTF guidelines on subsequent decision for biopsy would have likely been distributed equally among PSA-only and MRI groups given the temporal overlap of both cohorts as well as the focus of these recommendations having been on the role of PSA screening rather than the decision for biopsy among those with elevated PSA. Furthermore, several factors mitigate the impact of this policy shift on observed differences between groups, including study of the same providers prior to and after 2012 as well as the lack of corresponding changes in PSA-testing and biopsy guidelines set forth by the American Urological Association or American Cancer Society. The use of endorectal coil in our series may not be representative of current practice given improvements in mpMRI technology. However, use of endorectal coil in our series was based on limitations in the amount of signal needed to obtain high quality prostate imaging in the early phase of our MRI program. ${ }^{19}$ The limited follow-up of our study cohort is a limitation, particularly with respect to the consequences of missing csPCa in patients with negative MRI. All patients in our study, however, would reenter a PSA screening protocol and undergo repeat evaluation in the context of concerning PSA change with repeat MRI or standardized biopsy. This practice may mitigate, but not entirely eliminate, the risks of missed detection of csPCa in 
patients for whom initial biopsy was deferred due to a negative MRI result. Lastly, patients were from two independent but regionally-confined academic-community practices. Studies from centers across the country are needed to confirm generalizability.

In conclusion, with the growing body of level 1 evidence supporting mpMRI in the assessment of a man's risk of PCa, ${ }^{1,2,6}$ we demonstrate that when implemented in clinical practice, the use of mpMRI to triage patients with elevated PSA does indeed increase detection of csPCa in the context of reduced number of prostate biopsies. Although these improvements support a possible paradigm shift in the evaluation of patients with elevated PSA, we also recognize that the full impact of MRI to guide biopsy decision is not currently understood and will likely require a decade of follow-up to understand its clinical relevance. As such, our study results support the notion that in the short-term, use of MRI as a triage tool in men with elevated PSA offers opportunity to better diagnose and risk-stratify PCa than the status quo.

\section{References}

1. Ahmed HU, El-Shater Bosaily A, Brown LC, et al. Diagnostic accuracy of multi-parametric MRI and TRUS biopsy in prostate cancer (PROMIS): a paired validating confirmatory study. Lancet. 2017;389(10071):815-822.

2. Siddiqui MM, Rais-Bahrami S, Turkbey B, et al. Comparison of MR/ultrasound fusionguided biopsy with ultrasound-guided biopsy for the diagnosis of prostate cancer. JAMA. 2015;313(4):390-397.

This article is protected by copyright. All rights reserved 
3. Simmons LAM, Kanthabalan A, Arya M, et al. The PICTURE study: diagnostic accuracy of multiparametric MRI in men requiring a repeat prostate biopsy. Br J Cancer. 2017;116(9):1159-1165.

4. Turkbey B, Mani H, Shah V, et al. Multiparametric 3T prostate magnetic resonance imaging to detect cancer: histopathological correlation using prostatectomy specimens processed in customized magnetic resonance imaging based molds. J Urol. 2011;186(5):1818-1824.

5. Bibbins-Domingo K, Grossman DC, Curry SJ. The US Preventive Services Task Force 2017 Draft Recommendation Statement on Screening for Prostate Cancer: An Invitation to Review and Comment. JAMA. 2017;317(19):1949-1950.

6. Kasivisvanathan V, Rannikko AS, Borghi M, et al. MRI-Targeted or Standard Biopsy for Prostate-Cancer Diagnosis. N Engl J Med. 2018.

7. Moyer VA. Screening for prostate cancer: U.S. Preventive Services Task Force recommendation statement. Ann Intern Med. 2012;157(2):120-134.

8. Rastinehad AR, Waingankar N, Turkbey B, et al. Comparison of Multiparametric MRI Scoring Systems and the Impact on Cancer Detection in Patients Undergoing MR US Fusion Guided Prostate Biopsies. PLoS One. 2015;10(11):e0143404.

9. Epstein JI, Walsh PC, Carmichael M, Brendler CB. Pathologic and clinical findings to predict tumor extent of nonpalpable (stage T1c) prostate cancer. Jama. 1994;271(5):368-374.

10. Etzioni R, Gulati R, Falcon S, Penson DF. Impact of PSA screening on the incidence of advanced stage prostate cancer in the United States: a surveillance modeling approach. Med Decis Making. 2008;28(3):323-331.

11. Gulati R, Tsodikov A, Etzioni R, et al. Expected population impacts of discontinued prostate-specific antigen screening. Cancer. 2014;120(22):3519-3526.

12. Jemal A, Ma J, Siegel R, Fedewa S, Brawley O, Ward EM. Prostate Cancer Incidence Rates 2 Years After the US Preventive Services Task Force Recommendations Against Screening. JAMA Oncol. 2016;2(12):1657-1660. 
13. Bill-Axelson A, Holmberg L, Garmo H, et al. Radical prostatectomy or watchful waiting in early prostate cancer. N Engl J Med. 2014;370(10):932-942.

14. Hamdy FC, Donovan JL, Lane JA, et al. 10-Year Outcomes after Monitoring, Surgery, or Radiotherapy for Localized Prostate Cancer. N Engl J Med. 2016;375(15):1415-1424.

15. Grenabo Bergdahl A, Wilderang $U$, Aus $G$, et al. Role of Magnetic Resonance Imaging in Prostate Cancer Screening: A Pilot Study Within the Goteborg Randomised Screening Trial. Eur Urol. 2016;70(4):566-573.

16. Schoots IG, Roobol MJ, Nieboer D, Bangma CH, Steyerberg EW, Hunink MG. Magnetic resonance imaging-targeted biopsy may enhance the diagnostic accuracy of significant prostate cancer detection compared to standard transrectal ultrasound-guided biopsy: a systematic review and meta-analysis. Eur Urol. 2015;68(3):438-450.

17. Filson CP, Natarajan S, Margolis DJ, et al. Prostate cancer detection with magnetic resonance-ultrasound fusion biopsy: The role of systematic and targeted biopsies. Cancer. 2016;122(6):884-892.

18. Weinreb JC, Barentsz JO, Choyke PL, et al. PI-RADS Prostate Imaging - Reporting and Data System: 2015, Version 2. Eur Urol. 2016;69(1):16-40.

19. Turkbey B, Merino MJ, Gallardo EC, et al. Comparison of endorectal coil and nonendorectal coil T2W and diffusion-weighted MRI at 3 Tesla for localizing prostate cancer: correlation with whole-mount histopathology. J Magn Reson Imaging. 2014;39(6):1443-1448.

This article is protected by copyright. All rights reserved 


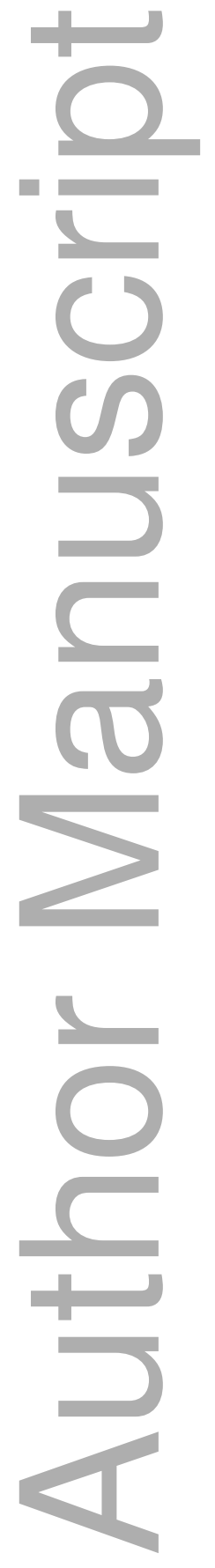

This article is protected by copyright. All rights reserved 
Table 1: Baseline participants' characteristics by era

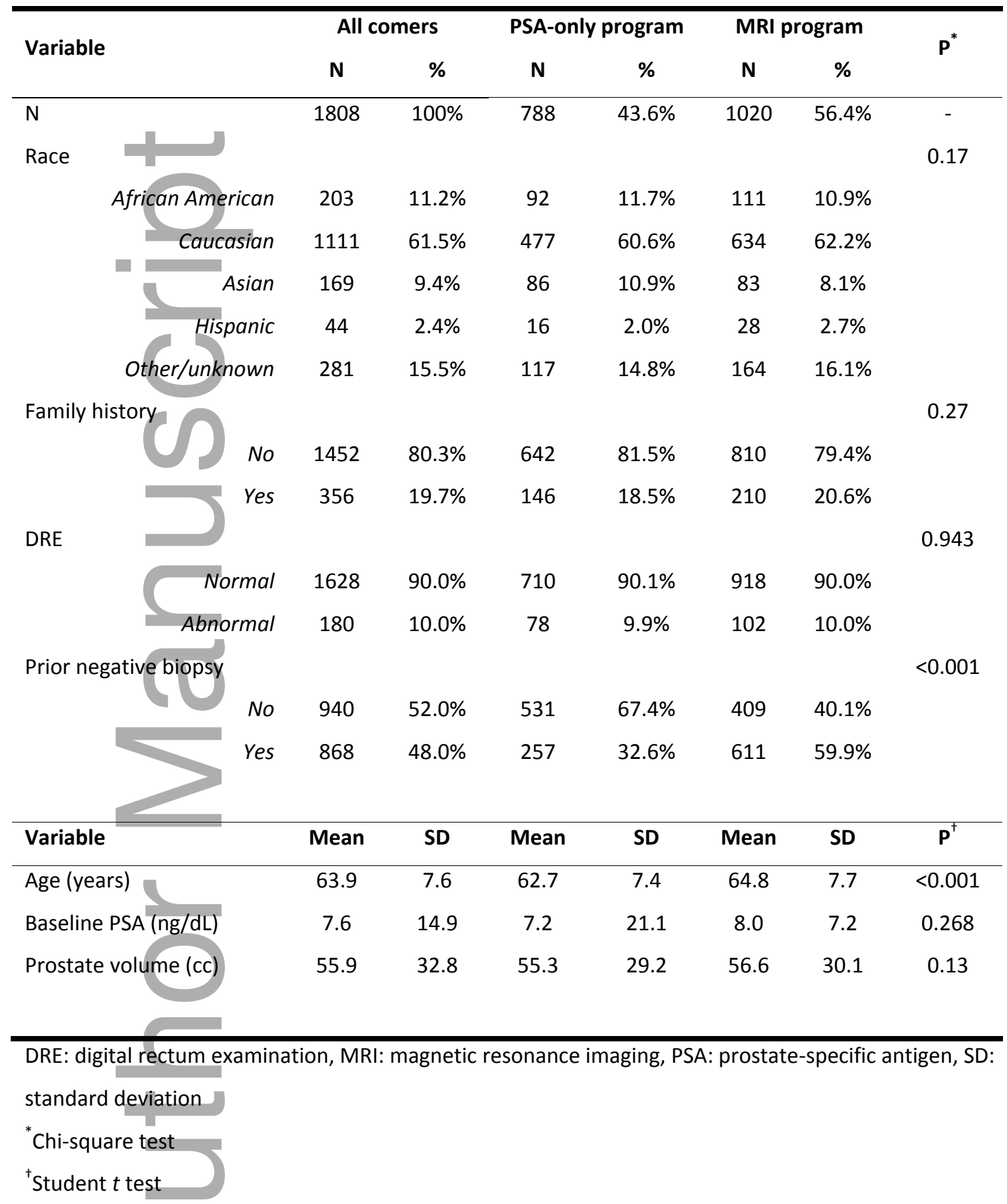

This article is protected by copyright. All rights reserved 
Table 2: Prostate cancer characteristics by group

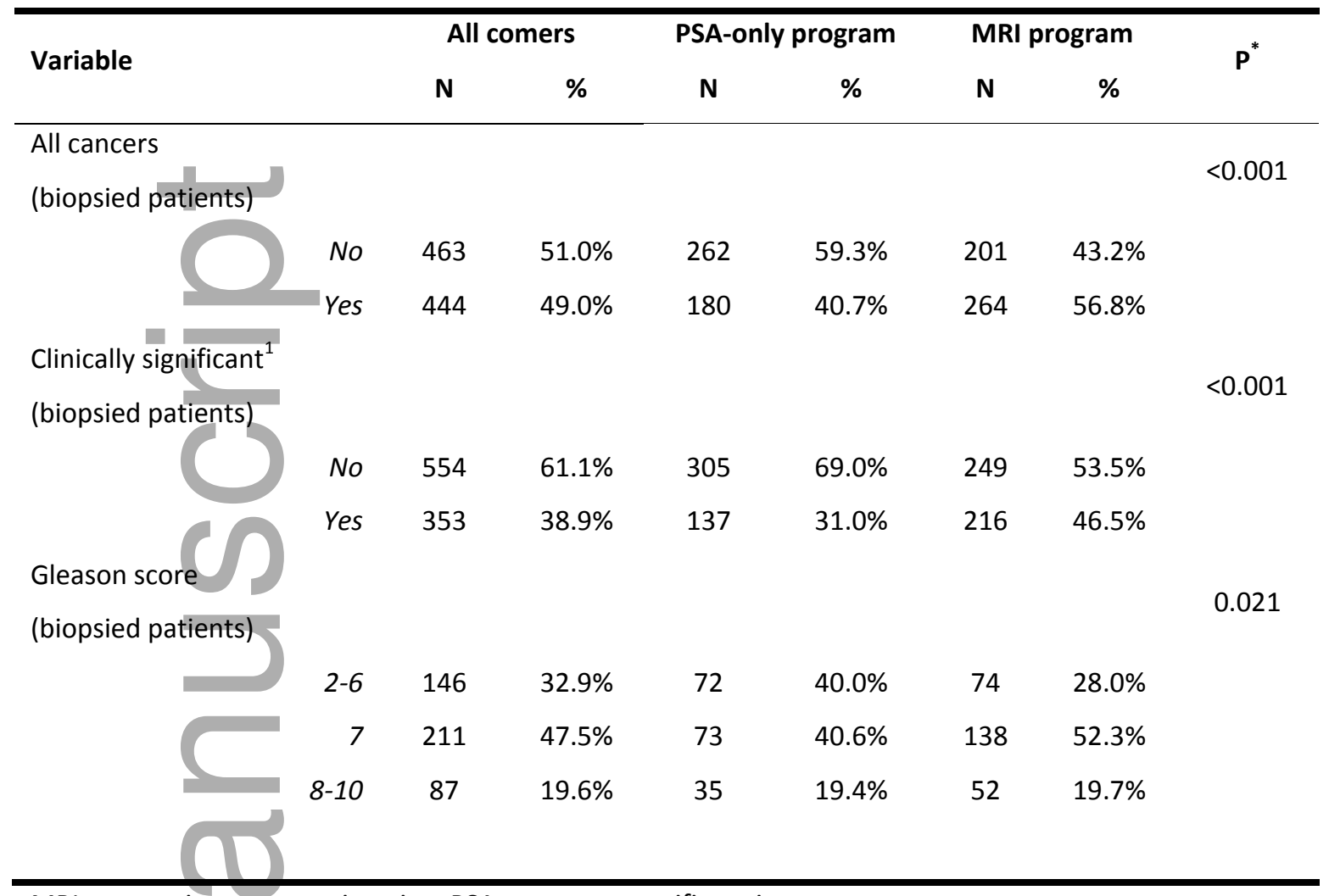

MRI: magnetic resonance imaging, PSA: prostate-specific antigen

${ }^{*}$ Chi-square test

${ }^{1}$ Gleason score $\geq 7$, Gleason score 6 with MRI-visible lesion volume $\geq 0.5 c c$, or Gleason score 6 with $>2$ positive cores and/or $>50 \%$ involvement of any core

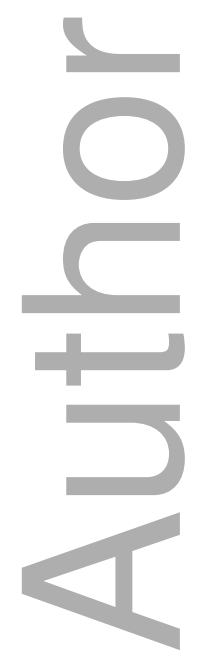

This article is protected by copyright. All rights reserved 
Table 3: Multivariable analysis of prostate cancer detection comparing MRI program to PSA-only program

\begin{tabular}{|c|c|c|c|c|c|c|c|}
\hline \multirow{2}{*}{ Prostate cancer } & & \multicolumn{3}{|c|}{ Univariable } & \multicolumn{3}{|c|}{ Multivariable $^{\mp}$} \\
\hline & & OR & $95 \% \mathrm{Cl}$ & $\mathbf{P}^{*}$ & OR & $95 \% \mathrm{Cl}$ & $\mathbf{P}^{*}$ \\
\hline All cancers (biopsied patients) & & 1.84 & $1.41-2.40$ & $<0.001$ & 1.74 & $1.29-2.35$ & $<0.001$ \\
\hline Clinically significant ${ }^{1}$ (biopsied patients & & 2.00 & $1.52-2.62$ & $<0.001$ & 2.04 & $1.48-2.80$ & $<0.001$ \\
\hline Prostate cancer $^{\mp}$ & & RRR & $95 \% \mathrm{Cl}$ & $\mathbf{P}^{+}$ & RRR & $95 \% \mathrm{Cl}$ & $\mathbf{P}^{+}$ \\
\hline Gleason score (biopsied patients) & $2-6$ & ref. & - & - & ref. & - & - \\
\hline & 7 & 1.86 & $1.21-2.87$ & 0.005 & 1.90 & $1.16-3.11$ & 0.010 \\
\hline & $8-10$ & 1.47 & $0.86-2.51$ & 0.164 & 1.58 & $0.83-2.99$ & 0.164 \\
\hline
\end{tabular}

OR: odds ratio, RRR: relative risk ratio

*Logistic regression

${ }^{\dagger}$ Multinomial logistic regression

${ }^{\ddagger}$ Analyses adjusted for: age, race, family history of prostate cancer, digital rectal exam, history of previous negative prostate biopsy and baseline prostate-specific antigen.

${ }^{1}$ Gleason score $\geq 7$, Gleason score 6 with MRI-visible lesion volume $\geq 0.5 \mathrm{cc}$, or Gleason score 6 with $>2$ positive cores and/or $>50 \%$ involvement of any core

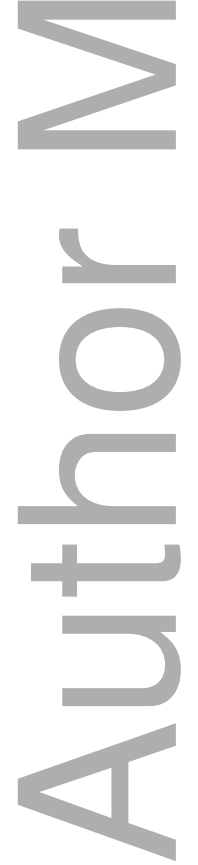

This article is protected by copyright. All rights reserved 
Table 4: Prostate cancer characteristics by biopsy type in patients who underwent MRI-guided fusion biopsy

\begin{tabular}{|c|c|c|c|c|c|c|c|}
\hline & \multicolumn{6}{|c|}{ Combined fusion and 12 -core (+ MRI) } & \multirow[t]{3}{*}{$\mathrm{P}^{*}$} \\
\hline & \multicolumn{2}{|c|}{ All } & \multicolumn{2}{|c|}{ Fusion Target } & \multicolumn{2}{|c|}{ 12-core } & \\
\hline & $\mathrm{N}$ & $\%$ & $\mathrm{~N}$ & $\%$ & $\mathrm{~N}$ & $\%$ & \\
\hline $\mathrm{N}$ & 324 & - & 324 & - & 324 & - & \\
\hline \multirow[t]{2}{*}{ All cancers } & & & & & & & 0.875 \\
\hline & 111 & $34.3 \%$ & 146 & $45.1 \%$ & 148 & $45.7 \%$ & \\
\hline 口 & 213 & $65.7 \%$ & 178 & $54.9 \%$ & 176 & $54.3 \%$ & \\
\hline \multirow[t]{3}{*}{ Clinically significant $^{1}$} & & & & & & & 0.022 \\
\hline & 147 & $45.4 \%$ & 161 & $49.7 \%$ & 190 & $58.6 \%$ & \\
\hline & 177 & $54.6 \%$ & 163 & $50.3 \%$ & 134 & $41.4 \%$ & \\
\hline Gleason score & & & & & & & 0.009 \\
\hline $2-6$ & 52 & $24.4 \%$ & 36 & $20.3 \%$ & 61 & $34.7 \%$ & \\
\hline & 118 & $55.4 \%$ & 107 & $60.5 \%$ & 84 & $47.7 \%$ & \\
\hline $8-10$ & 43 & $20.2 \%$ & 34 & $19.2 \%$ & 31 & $17.6 \%$ & \\
\hline
\end{tabular}

MRI: magnetic resonance imaging

${ }^{*}$ Chi-square test

${ }^{1}$ Gleason score $\geq 7$, Gleason score 6 with MRI-visible lesion volume $\geq 0.5 \mathrm{cc}$, or Gleason score 6 with $>2$ positive cores and/or $>50 \%$ involvement of any core

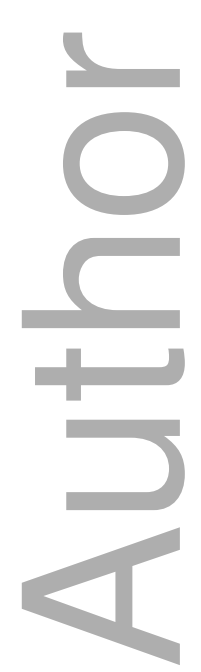

This article is protected by copyright. All rights reserved 